\title{
Probabilidad de obtener un título académico en Colombia
}

\section{Probability of obtaining an academic degree in Colombia}

\author{
Oscar Cerquera-Losada \\ Universidad Surcolombiana, Neiva, Colombia \\ Camilo Gómez-Segura \\ Universidad Surcolombiana, Neiva, Colombia \\ Libardo Rojas-Velásquez \\ Universidad ICESI, Cali, Colombia
}

\section{Resumen}

Objetivo: Determinar la probabilidad de obtener un determinado título académico en Colombia, dado un conjunto de variables que caracterizan a los individuos. Método: es un estudio cuantitativo desarrollado a través de un modelo econométrico de probabilidad Probit ordenado según cuatro categorías que corresponden a cada título académico respetivamente: ninguno, bachiller, técnico, universitario. Los datos provienen de la Gran Encuesta Integrada de Hogares (GEIH) del año 2018. Resultados: las mujeres tienen mayor probabilidad de tener un título de técnico y universitario que los hombres, los casados, los jefes de hogar, los que viven en la zona urbana y los que tienen vivienda propia, también presentan mayor probabilidad de tener un título de educación técnica y superior. Discusión y Conclusiones: las mujeres presentan mayores niveles de educación que los hombres; el nivel académico no necesariamente garantiza mayores probabilidades de conseguir empleo, pues los desempleados presentan mayores probabilidades de tener educación técnica y superior.

Palabras clave: Educación, efectos marginales, probabilidad, Probit ordenado, título académico.

\begin{abstract}
Objective: To determine the probability of obtaining a certain academic degree in Colombia, given a set of variables that characterize individuals. Method: It is a quantitative study developed through Probit probability econometric model, arranged into four categories corresponding to the following academic titles: none, secondary education, technical education, university. The data is gained from Comprehensive Household Survey (Gran Encuesta Integrada de Hogares - GEIH) of 2018. Results: Women are more likely to possess technical education and university degrees than men, married people, heads of household, those who live in urban areas and those owning their own homes. Discussion y Conclusion: Women achieve higher levels of education than men; the academic level does not necessarily guarantee a greater probability of getting a job, as people unemployed are more likely to have attained technical and university education.
\end{abstract}

Keywords: Education, marginal effects, probability, ordered Probit, academic title.
Open Access:

ISSN: $0124-2121$ E-ISSN: $2665-2420$

\author{
ARTÍ́CULO DE INVESTIGACIÓN \\ CIENTÍFICA \\ Copyright $(\subset$ \\ By Educación y Humanismo \\ Editor: \\ Dhayana Fernández Matos
}

Correspondencia: oscar.cerquera@usco.edu.co
Recibido: $30-09-2020$ Aceptado: 25-04-2020 En línea desde: $15-07-2021$ 


\section{Introducción}

La educación es un aspecto importante en el desarrollo económico de los países; en la literatura se reconocen al menos tres canales a través de los cuales la educación puede impactar el bienestar. Primero, autores como Lucas (1988), Mankiw, Romer y Weil (1992), consideran que la educación puede incrementar el capital humano de la fuerza laboral y por lo tanto la productividad. Segundo, Romer $(1986,1989)$ considera la educación como un promotor de la capacidad de innovación lo que impulsa la Investigación y el Desarrollo generando productos con valor agregado que impactan el sector productivo. $Y$ tercero, de acuerdo con Benhabib y Spiegel (1994), la educación facilita la difusión y transmisión de conocimiento necesario para procesar las nuevas tecnologías. Al ser la educación un elemento fundamental para el desarrollo de los países, su estudio se hace cada vez más necesario con el propósito de determinar cuáles son los elementos que favorecen un mayor desarrollo de la misma, teniendo clara la importancia que esta ejerce sobre el crecimiento y desarrollo económico de los pueblos (Becker, 1975).

El capital humano, factor indispensable para el proceso productivo, es importante a medida que mayor participación tenga; la forma de incentivar dicho factor es a través de la educación, la cual se debe concebir no sólo en número de años sino también en calidad y pertinencia. Según Lucas (1988), en muchos países del mundo el crecimiento económico es generado en mayor proporción por el capital humano, mientras más amplio y elevado es, mayor incidencia tiene sobre la producción.

La educación en Colombia ha sido un proceso que ha avanzado lentamente, pues se ha visto afectada desde tiempos remotos por distintos actores de la sociedad y se ha vinculado al desarrollo de muchos aspectos del país en los que se ha llevado una lucha o un proceso para permitirla. Desde un principio y aun en nuestros días, la educación ha sido un privilegio que se ha visto concentrado en ciertos grupos sociales (antes más notorio que ahora) (González, 1989). Desde la época colonial, la educación y el acceso a ella estaba establecido solo para los nobles y los religiosos, mientras que las mujeres no tenían derecho a estudiar. Más adelante se limita a las condiciones económicas y sociales y se ve como el Estado no contribuye al aporte de recursos para la educación.

De acuerdo con la Cepal (2019), Colombia es el segundo país de la región latinoamericana que presenta el coeficiente de GINI más alto con $50.8 \%$, después de Brasil $(51.3 \%)$. Esto implica que, a nivel interno, las desigualdades entre regiones son demasiado evidentes.

De acuerdo con un informe sobre la educación en Colombia desarrollado por la Organización para la Cooperación y el Desarrollo Económicos (OCDE, 2016), solo el 30\% de los jóvenes hace la transición de la escuela o colegio a la educación universitaria, y de estos, 
muchos desertarán antes de terminar. Las tasas de deserción anual en educación universitaria varían entre el $10.4 \%$ en las universidades y el $22.2 \%$ en las instituciones técnicas y tecnológicas; el 36\% de los jóvenes entre los 15 y los 19 años de edad en Colombia no están estudiando, esta cifra está por encima de los países de la OCDE que registran un promedio de $13 \%$. Por otro lado, el $19 \%$ de los jóvenes colombianos entre 15 y 19 años ni estudia, ni trabaja, ni recibe educación en comparación con el promedio de la OCDE del 7\% (OCDE, 2016).

Colombia presenta niveles de educación muy bajos en comparación con otros países. De acuerdo con la OCDE, sólo el $22 \%$ de las personas que están en un rango de edad entre los 25 y 64 años tienen un título universitario en Colombia, cifra por debajo del promedio de los países que pertenecen a la OCDE (38\%) (OCDE, 2019).

Por otro lado, según cifras de la Fundación Empresarios por la Educación, en el 2018, el $56 \%$ de los colombianos no completa la educación secundaria, y solo el $38 \%$ de los estudiantes que terminaron el bachillerato lograron inscribirse en la educación universitaria. El promedio de años de educación para Colombia es de 7.6 años, ocupando el puesto 119 de 187 países a nivel mundial, y el último lugar a nivel suramericano.

Tabla 1.

Porcentaje de individuos por título académico y clasificación laboral en 2018

\begin{tabular}{lrrr}
\hline \multicolumn{1}{c}{ Título } & PET & Ocupados & Desocupados \\
Ninguno & $54.4 \%$ & $45.4 \%$ & $32.6 \%$ \\
Bachiller & $29.1 \%$ & $32.4 \%$ & $43.6 \%$ \\
Técnico o tecnológico & $8.2 \%$ & $10.7 \%$ & $13.8 \%$ \\
Universitario & $5.9 \%$ & $8.0 \%$ & $8.4 \%$ \\
Posgrado & $2.4 \%$ & $3.6 \%$ & $1.7 \%$ \\
\hline
\end{tabular}

Fuente: Elaboración propia con base en datos de la GEIH (DANE, 2018)

De acuerdo con cifras de la Gran Encuesta Integrada de Hogares (GEIH) para el año 2018, del total de población colombiana en edad de trabajar ${ }^{1}$, el $54 \%$ no alcanzó ningún título académico, solo 3 de cada 10 poseen un título de bachiller, el $8.2 \%$ son técnicos o tecnólogos y el $5.9 \%$ universitarios. Con respecto a la población ocupada, la distribución es similar, el $45.4 \%$ de los ocupados en Colombia no tienen ningún título académico, el 32.4\% son bachilleres, y solo el $8 \%$ son universitarios. La mayor proporción de desocupados (43.6\%), presentan título de bachiller, el $32.6 \%$ no presentan ningún título y el $13.8 \%$ son técnicos o tecnólogos.

De acuerdo con Ferreyra (2017), las brechas de acceso a la educación universitaria se deben a que la gran mayoría de estudiantes en Colombia provienen de sectores de escasos

\footnotetext{
${ }^{1}$ La población en edad de trabajar está constituida por las personas mayores de 12 años en la zona urbana, y de 10 años en la zona rural.
} 
recursos y estos no alcanzan a graduarse del bachillerato o si se gradúan, su nivel académico está por debajo de la media, lo que limita la posibilidad de alcanzar un título de educación universitaria.

Alcanzar un determinado título universitario en Colombia, se está volviendo cada vez más complicado, de ahí la importancia de este trabajo, que busca determinar cuál es la probabilidad que tiene una persona para alcanzar algún título de educación, ya sea, título de bachiller, técnico y universitario, de acuerdo a diferentes características socioeconómicas de los individuos. Los datos provienen de la Gran Encuesta Integrada de Hogares (GEIH) para el año 2018.

Se estimó un modelo Probit ordenado, el cual permite que la variable dependiente tome más de dos categorías las cuales deben estar ordenadas; en este caso, se trabajará con cuatro categorías correspondientes a determinados niveles de la educación tales como ningún nivel, bachillerato, técnico y universitario.

No son muchos los estudios que se han realizado sobre este tema, Cameron y Hechman (1998), y Cameron y Taber (1994) son algunos de los estudios pioneros en la materia, quienes encuentran que el "entorno familiar" juega un papel fundamental en la decisión de finalizar los niveles educativos iniciados. Giovagnoli (2002) analizó el problema de la graduación y deserción solamente en los estudiantes universitarios de una cohorte de la carrera de Contador Público de la Universidad Nacional de Rosario, Argentina, utilizando modelos de duración que determinaron la probabilidad de que un estudiante se gradúe o deserte dado un conjunto de variables personales y socioeconómicas; la autora encuentra que el nivel de educación alcanzado por sus padres influye significativamente en la probabilidad de graduarse. Así mismo, si un estudiante empieza un trabajo durante el último año de carrera, tiene menor probabilidad de graduarse, las mujeres y los estudiantes solteros, también tienen mayor probabilidad de graduarse.

Osorio, Bolancé, y Caicedo (2012), estudiaron las características individuales y académicas que más se relacionan con la probabilidad de que un estudiante se gradué o no en la Universidad Pontificia Universidad Javeriana de Cali, Colombia. Los autores encontraron, con base en la estimación de modelos de probabilidad de supervivencia en tiempo discreto, que la deserción está más influenciada por características de tipo académico, mientras que la graduación está determinada por variables tanto personales como académicas. Por otra parte, Mendoza, Mendoza, y Romero (2014), realizaron un diagnóstico a la población de estudiantes de la Universidad Autónoma del Caribe, en Colombia, con el propósito de determinar los factores que condicionan la permanencia académica; la educación de los padres, el estado civil, las características socioeconómicas, son características que se relacionan positivamente con la permanencia y culminación del ciclo universitario.

Persoglia, Carella y Solari (2017), analizaron algunas características de los graduados de 
la Facultad de Ciencias Económicas de la Universidad Nacional de La Plata en Argentina. Estudiantes que egresaron de colegios privados, con padres de mayor nivel de educación formal, y con mayores promedios en la escuela secundaria, tienen mayor posibilidad de graduarse.

La literatura existente, generalmente se concentra en analizar los determinantes de que un estudiante, en un nivel específico de la educación, alcance su graduación, y los estudios se enfocan a nivel de programas e instituciones de educación secundaria y universitaria. El aporte de este trabajo radica en que se analiza los determinantes de que un individuo tenga un título de bachiller, técnico o tecnólogo, universitario, o simplemente no tenga ningún título académico. Este estudio se aplica a toda la población de Colombia, de acuerdo con los datos de la GEIH, analizando los cuatro niveles académicos ya descritos.

El presente artículo se organiza en cuatro partes. En la primera se abordó la introducción, en la cual se planteó el problema de investigación, se desarrollaron algunos antecedentes y se determinaron los propósitos de la investigación. En la segunda parte, se exponen la metodología y las herramientas necesarias para alcanzar los objetivos propuestos. En el siguiente apartado, se desarrollan los resultados. Y en la cuarta y última parte, se abordan las principales discusiones y conclusiones.

\section{Método}

Este artículo se clasifica en lo que se conoce como el enfoque cuantitativo, debido a que el propósito es determinar la probabilidad de que un individuo tenga un determinado título académico. Hernández, Cuevas, Leal y Mendoza (2016), plantean que, en el enfoque cuantitativo, el investigador utiliza sus diseños para analizar la certeza del problema planteado y aportar evidencias respecto a los lineamientos de la investigación, en este caso, determinar las variables que afectan la probabilidad de tener un título académico.

Además, este artículo se ajusta a los propósitos de la investigación no experimental, también conocida como investigación ex post facto, término que en latín significa, después de ocurridos los hechos. "La investigación no experimental es un tipo de investigación sistemática en la que el investigador no tiene control sobre las variables independientes porque ya ocurrieron los hechos o porque son intrínsecamente manipulables" (Kerlinger, 2002, p. 269). En este trabajo, los individuos ya se graduaron de un determinado nivel académico, es decir, ya el suceso tuvo lugar, por lo tanto, el propósito es explicar cuáles fueron los factores que determinaron que dicho evento (tener un título académico), ocurriera.

El diseño no experimental con enfoque cuantitativo hace referencia a un estudio en el que se manipulan intencionalmente una o más variables independientes (supuestas causasantecedentes), para analizar las consecuencias que la manipulación tiene sobre una o más 
variables dependientes (supuestos efectos-consecuentes), dentro de una situación de control. Particularmente se trabajó el diseño transeccional correlacional-causal que describe las relaciones entre dos o más categorías, conceptos o variables en un momento determinado, sea en términos de correlaciones, o en función de la relación causa-efecto (Hernández, Fernández y Baptista, 2010).

El propósito de este artículo es identificar las variables que caracterizan a la población colombiana y que determinan la probabilidad de que una persona tenga un determinado título académico. Para esto, se utilizó la base de datos suministrada por el Departamento Administrativo Nacional de Estadística (DANE), específicamente la base de datos de la GEIH en donde se encuentra información de carácter nacional sobre los diversos sectores. La información analizada corresponde al año 2018.

En la investigación se seleccionaron a todas las personas que respondieron la GEIH dentro de los grupos de cabecera y resto; se utilizó una muestra total de 348,807, y con el propósito de hacer inferencia estadística, para las estimaciones se utilizó el factor de expansión que por defecto contiene la base de datos. Es importante mencionar que el factor de expansión se aplica a los datos muestrales, y da a cada uno de los elementos de la muestra el peso representativo que le corresponde en el total de la población. Por tal razón, mediante su aplicación, se estiman numéricamente, en forma aproximada, las características de la población objetivo.

El primer paso realizado, consistió en caracterizar a la población estudiada con base en el análisis estadístico descriptivo. Esto permitió establecer un listado inicial de las variables incluidas en el estudio. En seguida, de estimó el modelo econométrico planteado, un modelo Probit Ordenado que permite determinar cuál es la probabilidad de que ocurra un conjunto determinado de eventos, en este caso, de tener un título académico ya sea a nivel de bachiller, técnico o tecnológico, universitario o ninguno. La diferencia entre estos modelos con respecto a los modelos clásicos de probabilidad es que permiten que la variable dependiente tome más de dos valores específicos, en este caso 4, uno por nivel académico alcanzado.

En el desarrollo de las estimaciones econométricas se utilizó el modelo Probit de respuesta múltiple ordenado. En este caso, la variable dependiente posee cuatro categorías de salida sujetas a ordenamiento, la primera hace referencia a un individuo sin ningún título académico logrado, la segunda y tercera indican los títulos de bachiller y técnico y/o tecnólogo, respectivamente, y la cuarta se refiere al título universitario.

$$
y_{i}=X \beta+\varepsilon
$$

Asumiendo a $y_{i}$ como una función de los diferentes niveles de títulos, $X \beta$ las variables y los parámetros a estimar, y $\varepsilon$ el error. No obstante, $y_{i}$ es una variable latente no observada, debido a que solo conocen los resultados discretos. En este caso, estos resultados son observados a través de la siguiente regla: 
título $=1$ (ningún título)

título $=2$ (Bachiller)

título $=2$ (técnico y tecnólogo $)$

título $=1$ (universitario) si $y_{i} \leq \Delta_{1}$

si $\Delta_{1}<y_{i} \leq \Delta_{2}$

Sí $\varepsilon$ se distribuye normal, las categorías de la ecuación anterior implican un modelo Probit ordenado, que se representa de la siguiente manera:

$$
\begin{gathered}
\operatorname{Prob}(\eta=1)=\operatorname{Prob}\left(\varepsilon \leq \Delta_{1}-X \beta\right)=\Phi\left(\Delta_{1}-X \beta\right) \\
\operatorname{Prob}(\eta=2)=\operatorname{Prob}\left(\Delta_{1}-X \beta<\varepsilon \leq \Delta_{2}-X \beta\right)=\Phi\left(\Delta_{2}-X \beta\right)-\Phi\left(\Delta_{1}-X \beta\right)(2) \\
\operatorname{Prob}(\eta=3)=\operatorname{Prob}\left(\Delta_{2}-X \beta<\varepsilon \leq \Delta_{3}-X \beta\right)=\Phi\left(\Delta_{3}-X \beta\right)-\Phi\left(\Delta_{2}-X \beta\right) \\
\operatorname{Prob}(\eta=4)=\operatorname{Prob}\left(\varepsilon>\Delta_{3}-X \beta\right)=1-\Phi\left(\Delta_{3}-X \beta\right)
\end{gathered}
$$

Nótese que $\Delta_{1}, \Delta_{2}$, y $\Delta_{3}$ son puntos de corte y se calculan con el resto de estimadores. La función de verosimilitud del modelo multinomial ordenado se deriva como:

$$
\begin{array}{lcc}
l\left(\beta, \Delta_{1}, \Delta_{2}, \Delta_{3}\right)=\sum_{y_{i}=1} & \log \left(\Phi\left(\Delta_{1}-X \beta\right)\right)+\sum_{y_{i}=2} & \log \log \left(\Phi\left(\Delta_{2}-X \beta\right)-\Phi\left(\Delta_{1}-\right.\right. \\
X \beta))+\sum_{y_{i}=3} & \log \log \left(\Phi\left(\Delta_{3}-X \beta\right)-\Phi\left(\Delta_{2}-X \beta\right)\right)+\sum_{y_{i}=4} \quad \log \left(1-\Phi\left(\Delta_{3}-X \beta\right)\right)
\end{array}
$$

En donde $\Phi$ es la función de distribución acumulada de los errores $\varepsilon$, de la cual depende la especificación del modelo, que en este caso, por ser un Probit ordenado, presenta una distribución normal estándar.

$$
\Phi=\frac{1}{\sqrt{2 \pi}} e^{\frac{-X_{i} \beta^{2}}{2}}
$$

De acuerdo con Cameron y Trivedi (2005), los parámetros estimados de la regresión de cualquier modelo de probabilidad no se pueden analizar en términos de magnitudes, solo se puede estudiar el signo. Para determinar el aumento de la probabilidad en una determinada categoría dado la variación en una de las variables explicativas, se requiere estimar los efectos marginales:

$$
\begin{array}{r}
\frac{\partial P r\left(y_{i}=1\right)}{\partial x_{k}}=\Phi^{\prime}\left(\Delta_{1}-X \beta\right) \beta_{k} \\
\frac{\partial P r\left(y_{i}=2\right)}{\partial x_{k}}=\Phi^{\prime}\left(\Delta_{2}-X \beta\right) \beta_{k}-\Phi^{\prime}\left(\Delta_{1}-X \beta\right) \beta_{k}
\end{array}
$$




$$
\begin{gathered}
\frac{\partial \operatorname{Pr}\left(y_{i}=3\right)}{\partial x_{k}}=\Phi^{\prime}\left(\Delta_{3}-X \beta\right) \beta_{k}-\Phi^{\prime}\left(\Delta_{2}-X \beta\right) \beta_{k} \\
\frac{\partial \operatorname{Pr}\left(y_{i}=4\right)}{\partial x_{k}}=\Phi^{\prime}\left(1-\Delta_{3}-X \beta\right) \beta_{k}
\end{gathered}
$$

Como el número de categorías es mayor a dos, el modelo probabilístico ordenado generalizado es equivalente a una serie de regresiones probabilísticas binarias, donde las categorías de la variable dependiente estarían combinadas (Williams, 2006). En este caso, la combinación de categorías indica que la categoría ningún título (categoría base) es contrastada con las demás categorías, categoría título de bachiller, título técnico y tecnológico, y título universitario.

El modelo teórico que subyace a esta metodología es la teoría de capital humano desarrollada por Becker (1993), el cual sostiene que el nivel de inversión en capital humano de cada individuo emana de un proceso de optimización en el que se igualan los beneficios y costos marginales de educarse. Hanushek (1986), también hace aportes interesantes en esta materia con la teoría de la función de producción educativa, la cual establece que la economía puede analizar la educación con un enfoque de insumo-producto, es decir, un marco en el cual el logro académico es determinada por una serie de factores personales, familiares e institucionales ${ }^{2}$.

\section{Resultados}

\section{Análisis estadístico Descriptivo}

El análisis estadístico descriptivo permite caracterizar la muestra objeto de estudio, estableciendo el porcentaje de participación de los individuos para cada título de nivel educativo alcanzado en función de las características personales, del hogar y socioeconómicos establecidos en este artículo.

La tabla 2 presenta el porcentaje de individuos según las características personales en función del título de nivel educativo que han obtenido durante el año 2018.

Tabla 2

Porcentaje de individuos según las características personales para cada título de nivel educativo en el año 2018

Título \%

\begin{tabular}{lllll}
\hline Características Personales & Ninguno & Bachiller & Técnico & Universitario \\
\hline
\end{tabular}

${ }^{2}$ Para una aplicación de este enfoque ver Cerquera, Cano y Gómez (2016) 


$\begin{array}{crrrr}12-17 \text { años } & 57.40 & 42.05 & 0.54 & 0.01 \\ 18-26 \text { años } & 5.06 & 70.72 & 16.63 & 7.60 \\ 27-59 \text { años } & 2.15 & 52.79 & 20.02 & 25.05 \\ 60 \text { o más años } & 2.53 & 52.54 & 11.24 & 33.69\end{array}$

Género

\begin{tabular}{cccccc} 
& Hombre & 5.69 & 59.68 & 15.79 & 18.84 \\
Estado Civil & Mujer & 5.08 & 55.20 & 19.17 & 20.54 \\
& & & & & \\
& Unión libre & 3.75 & 62.79 & 19.30 & 14.16 \\
& Casado & 1.58 & 47.23 & 19.03 & 32.16 \\
& Separado & 3.15 & 57.35 & 19.38 & 20.11 \\
& Viudo & 3.01 & 59.98 & 14.95 & 22.06 \\
& Soltero & 9.71 & 58.91 & 15.13 & 16.25 \\
\hline
\end{tabular}

Fuente: Elaboración propia con base en datos de la GEIH (DANE, 2018)

Se logra evidenciar que existen diferencias en cada una de las variables de las características personales con respecto al título educativo. Con respecto a la edad, el $57.4 \%$ de la población con edad entre 12-17 años, no posee ningún título académico y el $42.05 \%$ tiene un título bachiller. Con respecto a las personas en edades entre 18 y 26 , el $70.72 \%$, cuenta con un título de Bachiller, el $16.63 \%$ tiene un título de Técnico y tan sólo el $7.60 \%$ ha obtenido un título educativo de nivel universitario. En los dos últimos grupos de edad, se concentra la mayor cantidad de universitarios con respecto a los demás grupos etarios, $25.05 \%$ y $33.69 \%$, respectivamente, sin embargo, en su mayoría las personas poseen título de bachiller en ambas categorías.

Con relación al género, las mujeres presentan una mayor proporción de individuos con título universitario (20.54\%) y técnico (19.27\%), por encima de los hombres, el $55.2 \%$ del total de mujeres son bachilleres. Con respecto a los hombres, el $59.68 \%$ son bachilleres y el $18.84 \%$ poseen un título universitario.

En cuanto al estado civil de la persona, se encuentra que los casados tienen mayor proporción en el nivel universitario, dentro de ese grupo el $32.16 \%$ cuenta con un título de educación universitario, $19.03 \%$ poseen un título de técnico, el $47.23 \%$ alcanzó un título de bachiller. En el caso de los que viven en unión libre, el 62.79\% tiene un título de educación Bachiller y el $14.16 \%$ ha alcanzado el título de educación universitario. En general, en todos los tipos de estado civil, prevalecen individuos con título de bachiller. El grupo de los solteros son los que mayor proporción de personas tienen sin ningún título, mientras que los viudos son quienes menor proporción presentan en el nivel técnico.

En la tabla 3 se presenta el porcentaje de individuos por título académico según las características del hogar, de nuevo para el año 2018. 
Tabla 3

Porcentaje de individuos según las características del hogar para cada título de nivel educativo en el año 2018

\begin{tabular}{crrrr}
\cline { 2 - 5 } & \multicolumn{5}{c}{ Título \% } \\
\hline Características del Hogar & Ninguno & Bachiller & \multicolumn{1}{c}{ Técnico } & Universitario \\
\hline Personas en el Hogar & & & & \\
1-7 personas & 5.20 & 56.74 & 17.71 & 20.35 \\
8-14 personas & 8.42 & 68.09 & 14.83 & 8.67 \\
15-21 personas & 8.94 & 77.29 & 9.66 & 4.11 \\
22-28 personas & 9.09 & 63.64 & 22.73 & 4.55 \\
Computador & & & & \\
Tiene Computador & 3.81 & 47.98 & 18.52 & 29.69 \\
No tiene Computador & 6.89 & 66.54 & 16.61 & 9.96 \\
Acceso a Internet & & & & \\
Con Acceso & 4.02 & 50.88 & 18.35 & 26.74 \\
Sin Acceso & 7.47 & 67.48 & 16.32 & 8.74 \\
\hline
\end{tabular}

Fuente: Elaboración propia con base en datos de la GEIH (DANE, 2018)

En cuanto al número de personas que conforman el hogar, se observa una relación inversa con respecto al título universitario, a mayor número de individuos que habiten en el hogar, menor va a ser el porcentaje de individuos que obtengan un título universitario. En hogares que viven de 1 a 7 personas, el $56.74 \%$ son bachilleres y el $20.35 \%$ han alcanzado un título educativo universitario. En el grupo de 15 a 21 años, existe la mayor proporción de personas con título de bachiller (77.29\%). Las herramientas educativas como las tecnológicas son un componente importante en logro educativo. De acuerdo con los resultados encontrados, los que tienen computador, el $47.98 \%$ ha obtenido un título bachiller, en contraste a los que no tienen computador donde el $66.54 \%$ poseen este título; en el caso del título universitario, de los que tienen computador, el $29.69 \%$ lo ha alcanzado, y sólo el $9.96 \%$ de los que no tienen computador ha obtenido el mismo título. En cuanto al acceso a internet, el 50.88\% tiene un título Bachiller, el $18.35 \%$ un título técnico y el $26.74 \%$ ha obtenido el título universitario.

Finalmente, la tabla 4 presenta el porcentaje de individuos según las características socioeconómicas en función del título de nivel educativo que han obtenido durante el periodo de estudio.

\section{Tabla 4}

Porcentaje de individuos según las características socioeconómicas para cada título de nivel educativo en el año 2018.

\begin{tabular}{lrrrrr} 
& \multicolumn{5}{c}{ Título \% } \\
\cline { 3 - 6 } \multicolumn{1}{l}{ Características Socioeconómicas } & Ninguno & Bachiller & Técnico & Universitario \\
\hline Estrato & & & & & \\
& Bajo & 6.61 & 63.75 & 17.92 & 11.72 \\
& Medio & 3.28 & 47.43 & 17.47 & 31.82 \\
& Alto & 3.38 & 33.24 & 7.40 & 55.98
\end{tabular}




\begin{tabular}{|c|c|c|c|c|}
\hline Urbana & 5.10 & 56.78 & 17.80 & 20.32 \\
\hline Rural & 11.65 & 69.85 & 11.78 & 6.72 \\
\hline \multicolumn{5}{|l|}{ Oficio } \\
\hline Trabaja & 2.35 & 52.31 & 20.42 & 24.92 \\
\hline Busca trabajo & 2.35 & 50.62 & 21.88 & 25.15 \\
\hline Estudia & 23.30 & 68.80 & 6.27 & 1.63 \\
\hline Actividades del Hogar & 3.80 & 65.55 & 17.25 & 13.40 \\
\hline Incapacitado para Trabajar & 3.38 & 68.73 & 9.35 & 18.54 \\
\hline Otra Actividad & 2.95 & 55.02 & 14.83 & 27.20 \\
\hline
\end{tabular}

Fuente: Elaboración propia con base en datos de la GEIH (DANE, 2018)

El nivel socioeconómico medido a través del estrato, indica una relación positiva con la formación académica. Con respecto a los individuos de estrato bajo (estrato 1 y 2), el $63.75 \%$ cuentan con un título de bachiller, el $17.92 \%$ con un título técnico y el $11.72 \%$ ha obtenido un título de educación universitaria; en las personas de estrato medio, el $31.8 \%$ son universitario y el $17.47 \%$ poseen un título técnico. En la población de estrato alto, se presenta la menor proporción de bachilleres (33.24\%), y la mayor proporción de universitarios (55.98\%) que, sumando los técnicos, alcanza la importante cifra de $67.4 \%$ de individuos con algún título académico en el nivel superior.

En cuanto a la zona en que reside el individuo, los resultados son los esperados; el $11.65 \%$ de los que viven en la zona rural no posee ningún título, el $69.85 \%$ tiene un título de bachiller, sólo el $6.72 \%$ ha logrado un título universitario; el acceso a niveles de educación más altos es más limitado en estas zonas. En contraste con la zona urbana, en donde el $56.78 \%$ cuenta con un título bachiller, el $17.8 \%$ ha alcanzado un título técnico y el $20.32 \%$, posee un título universitario.

Finalmente, con respecto al oficio u ocupación de los individuos, del total de individuos que actualmente están trabajando, el $52.31 \%$ tiene un título de bachiller, el $20.42 \%$ un título técnico y un $24.92 \%$ un título universitario, siendo uno de los grupos de ocupados con más altos niveles de educación universitaria. Por otro lado, el $25.15 \%$ de los que buscan trabajo ya poseen un título universitario, aspecto preocupante, y el $21.88 \%$ un título técnico. Otro dato relevante es que, del total de personas que se dedican a las actividades del hogar, el $13.4 \%$ y el $17.25 \%$ poseen títulos de técnico y universitario, respectivamente, que por lo general son mujeres, las cuales generalmente asumen la responsabilidad del cuidado, en mayor medida, de los hijos y los quehaceres del hogar, incluso aun después de participar en el mercado laboral.

\section{Modelo Econométrico}

Con el propósito de establecer cuál es la probabilidad de que una persona en Colombia obtenga un determinado título educativo, se utilizó un modelo Probit Ordenado. Para tal fin, 
se estimó la probabilidad de que una persona en Colombia logre un mayor nivel educativo con respecto a otro, considerando las características personales, socioeconómicas y del hogar.

La variable dependiente se encuentra clasificada en cuatro categorías, en donde cada una corresponde a un nivel académico respectivo, ningún título, bachiller, técnico y universitario. Las variables independientes se encuentran agrupadas en tres conjuntos de variables, características personales, del hogar y socioeconómicas.

La primera estimación, observada en la tabla 5, corresponde al modelo Probit ordenado corregido por heterocedasticidad con errores estándar robustos, donde solamente se puede hacer énfasis, al menos por ahora, en los signos y la significancia estadística de los parámetros. En todos los casos las variables son estadísticamente significativas. El signo negativo en las variables significa que existe una menor probabilidad de alcanzar un nivel educativo más alto, mientras que un signo positivo indica que existe una mayor probabilidad de tener un título académico más alto. En la estimación se registran tres intercepto lo que indica que la variable dependiente tiene cuatro categorías.

En términos generales, las relaciones de las estimaciones muestran los resultados esperados. Con respecto a la edad, a mayor edad, es más probable que el individuo alcance títulos académicos de mayor nivel. Los hombres (la variable género es una variable binaria donde 1 es igual a hombre) tienen menos probabilidad de tener mayores títulos de formación que las mujeres, al igual que los que están actualmente estudiando; un mayor número de miembros por hogar también se relaciona con una menor probabilidad de alcanzar mayores niveles académicos.

Los individuos que tienen computador en casa, acceso a internet, tienen vivienda propia y viven en la zona urbana, presentan una mayor probabilidad de tener mayores títulos de formación académica. Del mismo modo, los casados, los jefes de hogar, los que pertenecen al régimen contributivo y los que son hijos, también presentan una mayor probabilidad de alcanzar títulos de educación más altos. Los que están trabajando y los que están desempleados, también presentan mayores probabilidades de tener mayores títulos académicos, en el caso de los desempleados esto indica que, aun alcanzando los mayores niveles formación académica, la consecución de un empleo no está garantizada. Finalmente, a mayor estrato socioeconómico, mayor es la probabilidad de alcanzar títulos académicos de mayor nivel.

Tabla 5

Modelo Probit Ordenado

\begin{tabular}{|c|c|c|}
\hline Variables & Coeficientes & $\begin{array}{c}\text { Error } \\
\text { Estándar }\end{array}$ \\
\hline Edad & $0.0007 * * *$ & 0.0001 \\
\hline
\end{tabular}




\begin{tabular}{lrr} 
Hombre & $-0.2332 * * *$ & 0.0047 \\
Urbano & $0.1476 * * *$ & 0.0115 \\
Personas en el Hogar & $-0.0411^{* * *}$ & 0.0019 \\
Internet & $0.1595^{* * *}$ & 0.0055 \\
Computador & $0.2940 * * *$ & 0.0052 \\
Estrato & $0.1658^{* * *}$ & 0.0024 \\
Casado & $0.0279 * * *$ & 0.0053 \\
Jefe del Hogar & $0.0911^{* * *}$ & 0.0058 \\
Contribuyente & $0.3569 * * *$ & 0.0051 \\
Hijos & $0.0814 * * *$ & 0.0065 \\
Vivienda Propia & $0.1131 * * *$ & 0.0046 \\
Trabajador & $0.3048 * * *$ & 0.0054 \\
Desempleado & $0.6272 * * *$ & 0.0127 \\
Estudiante & $-1.0576 * * *$ & 0.0093 \\
\hline Pseudo R2 & 0.1182 & \\
\hline Número de Observaciones & 295.449 & \\
\multicolumn{1}{c}{ Elaboración propia con base en datos de la GEIH (DANE, 2018) } \\
Nota: ***, $* *, *$ denotes $1 \%, 5 \%$, and 10\% Significance level, respectively.
\end{tabular}

De acuerdo con Connelly et al (1989), en este tipo de modelos es común encontrar bajas medidas de bondad de ajuste. En nuestro caso, el Pseudo $\mathrm{R}^{2}$ fue de $11.82 \%$. No obstante, la bondad de ajuste provee información parcial que puede considerarse en el contexto de la teoría que sustenta el análisis, la investigación pasada y los parámetros estimados en el modelo que se está planteando (Long y Freese 2001).

Para poder analizar la magnitud de los parámetros, se estiman los efectos marginales, los cuales indican cómo los cambios marginales de una unidad afectan el cambio en la probabilidad predicha de alcanzar un determinado título; por lo tanto, se estiman los efectos marginales para cada una de las cuatro categorías incorporadas en el modelo (ningún título, bachiller, técnico, universitario). La tabla 6 muestra los efectos marginales para cada una de las variables explicativas con respecto a la media, es decir, manteniendo las variables fijas en sus medias.

Tabla 6

Efectos Marginales para cada Categoría de la Variable

\begin{tabular}{lcccc} 
& \multicolumn{4}{c}{ Título Obtenido } \\
\hline Variables & Ninguno & Bachiller & Técnico & Universitario \\
\hline \multirow{2}{*}{ Edad } & $-0.0001^{* * *}$ & $-0.0002^{* * *}$ & $0.0001^{* * *}$ & $0.0001^{* * *}$ \\
& $(0.0000)$ & $(0.0001)$ & $(0.0000)$ & $(0.0000)$ \\
Hombre & $0.0151^{* * *}$ & $0.0675^{* * *}$ & $-0.0377 * * *$ & $-0.0449 * * *$ \\
\multirow{2}{*}{ Urbano } & $(0.0003)$ & $(0.0013)$ & $(0.0007)$ & $(0.0009)$ \\
& $-0.0095^{* * *}$ & $-0.0427^{* * *}$ & $0.0238^{* * *}$ & $0.0284 * * *$
\end{tabular}




\begin{tabular}{|c|c|c|c|c|}
\hline & (0.0007) & (0.0033) & $(0.0018)$ & $(0.0022)$ \\
\hline Personas en el Hogar & $\begin{array}{c}0.0026 * * * \\
(0.0001)\end{array}$ & $\begin{array}{c}0.0119 * * * \\
(0.0005)\end{array}$ & $\begin{array}{c}-0.0066 * * * \\
(0.0003)\end{array}$ & $\begin{array}{c}-0.0079 * * * \\
(0.0003)\end{array}$ \\
\hline Internet & $\begin{array}{c}-0.0103 * * * \\
(0.0003)\end{array}$ & $\begin{array}{c}-0.0462 * * * \\
(0.0016)\end{array}$ & $\begin{array}{c}0.0258^{* * *} \\
(0.0008)\end{array}$ & $\begin{array}{c}0.0307 * * * \\
(0.0010)\end{array}$ \\
\hline Computador & $\begin{array}{c}-0.0191 * * * \\
(0.0003)\end{array}$ & $\begin{array}{c}-0.0851 * * * \\
(0.0015)\end{array}$ & $\begin{array}{c}0.0475 * * * \\
(0.0008)\end{array}$ & $\begin{array}{c}0.0566 * * * \\
(0.0010)\end{array}$ \\
\hline Estrato & $\begin{array}{c}-0.0107 * * * \\
(0.0001)\end{array}$ & $\begin{array}{c}-0.0480 * * * \\
(0.0007)\end{array}$ & $\begin{array}{c}0.0268 * * * \\
(0.0004)\end{array}$ & $\begin{array}{c}0.0319 * * * \\
(0.0004)\end{array}$ \\
\hline Casado & $\begin{array}{c}-0.0018^{* * *} \\
(0.0003)\end{array}$ & $\begin{array}{c}-0.0080 * * * \\
(0.0015)\end{array}$ & $\begin{array}{c}0.0045 * * * \\
(0.0008)\end{array}$ & $\begin{array}{l}0.0053 * * * \\
(0.0010)\end{array}$ \\
\hline Jefe del Hogar & $\begin{array}{c}-0.0059 * * * \\
(0.0003)\end{array}$ & $\begin{array}{c}-0.0263 * * * \\
(0.0016)\end{array}$ & $\begin{array}{c}0.0147 * * * \\
(0.0009)\end{array}$ & $\begin{array}{l}0.0175 * * * \\
(0.0011)\end{array}$ \\
\hline Contribuyente & $\begin{array}{c}-0.0231 * * * \\
(0.0003)\end{array}$ & $\begin{array}{c}-0.1033 * * * \\
(0.0015)\end{array}$ & $\begin{array}{c}0.0577 * * * \\
(0.0008)\end{array}$ & $\begin{array}{c}0.0687 * * * \\
(0.0009)\end{array}$ \\
\hline Hijos & $\begin{array}{c}-0.0052 * * * \\
(0.0004)\end{array}$ & $\begin{array}{c}-0.0235 * * * \\
(0.0018)\end{array}$ & $\begin{array}{l}0.0131 * * * \\
(0.0010)\end{array}$ & $\begin{array}{c}0.0156 * * * \\
(0.0012)\end{array}$ \\
\hline Vivienda Propia & $\begin{array}{c}-0.0073 * * * \\
(0.0003)\end{array}$ & $\begin{array}{c}-0.0327 * * * \\
(0.0013)\end{array}$ & $\begin{array}{c}0.0183 * * * \\
(0.0007)\end{array}$ & $\begin{array}{c}0.0217 * * * \\
(0.0009)\end{array}$ \\
\hline Trabajador & $\begin{array}{c}-0.0198 * * * \\
(0.0003)\end{array}$ & $\begin{array}{l}-0.0882 * * * \\
(0.0015)\end{array}$ & $\begin{array}{c}0.0493 * * * \\
(0.0009)\end{array}$ & $\begin{array}{c}0.0587 * * * \\
(0.0010)\end{array}$ \\
\hline Desempleado & $\begin{array}{c}-0.0407 * * * \\
(0.0008)\end{array}$ & $\begin{array}{l}-0.1816 * * * \\
(0.0037)\end{array}$ & $\begin{array}{c}0.1015^{* * *} \\
(0.0021)\end{array}$ & $\begin{array}{c}0.1208 * * * \\
(0.0024)\end{array}$ \\
\hline Estudiante & $\begin{array}{c}0.0687 * * * \\
(0.0007)\end{array}$ & $\begin{array}{c}0.3062 * * * \\
(0.0028)\end{array}$ & $\begin{array}{c}-0.1711 * * * \\
(0.0016)\end{array}$ & $\begin{array}{c}-0.2038^{* * *} * \\
(0.0018)\end{array}$ \\
\hline $\begin{array}{l}\text { Probabilidad de Tener un } \\
\text { Título }\end{array}$ & $2.83 \%$ & $65.80 \%$ & $19.97 \%$ & $11.38 \%$ \\
\hline
\end{tabular}

Elaboración propia con base en datos de la GEIH (DANE, 2018)

Nota: $* * *, * *, *$ denotes $1 \%, 5 \%$, and $10 \%$ significance level, respectively.

Un individuo con un año adicional de edad presenta un $0.01 \%$ menos de probabilidad de no tener ningún título académico, un $0.02 \%$ menos de probabilidad de tener un título de bachiller, un $0.012 \%$ más de probabilidad de tener un título técnico y un $0.014 \%$ más de probabilidad de alcanzar un título universitario. La edad afecta positivamente la probabilidad de tener títulos de educación de mayores niveles.

Con respecto al género, los hombres tienen una mayor probabilidad (1.5\% mayor) de no tener ningún título académico con respecto a las mujeres. Así mismo, los hombres tienen un $3.77 \%$ menos de probabilidad que las mujeres de tener un título de educación terciaria, y un $4.49 \%$ menos de probabilidad de alcanzar un título universitario. Es decir, los hombres tienen mayor probabilidad que las mujeres de alcanzar bajos niveles académicos, mientras que las mujeres tienen mayor probabilidad que los hombres de tener títulos académicos de mayor nivel.

En cuanto al estrato socioeconómico, un estrato adicional, representa un $1.07 \%$ menos probabilidad de no tener ningún título académico, un $4.6 \%$ menos de probabilidad de ser bachiller, un $0.45 \%$ más de probabilidad de tener un título técnico, y un $3.19 \%$ más de probabilidad de tener un título universitario. El estrato es importante en la formación del nivel técnico y universitario. Sí el individuo pertenece al régimen contributivo, tiene un 
$2.31 \%$ menos de probabilidad, que los que pertenecen al régimen subsidiado, de no tener ningún título académico, un $10.3 \%$ menos de probabilidad de ser bachiller, un $5.7 \%$ más de probabilidad de ser un técnico, y un $6.8 \%$ más de probabilidad de tener un título universitario. Quienes tienen vivienda propia, tienen un 3.2\% menos de probabilidad de tener un título bachiller, y un $2.1 \%$ más de probabilidad de tener un título universitario.

Los jefes de hogar tienen un $2.6 \%$ menos de probabilidad de tener un título de bachiller, y un $1.75 \%$ más de probabilidad de tener un título universitario. Los casados tienen un $0.18 \%$ menos de probabilidad de no tener ningún título académico, y un $0.5 \%$ más de probabilidad de ser universitario. Los desempleados, tienen un $4 \%$ menos de probabilidad de no tener ningún título académico, un $18.1 \%$ menos de probabilidad de tener un título de bachiller, un $10.15 \%$ más de probabilidad de tener un título técnico y un $12.08 \%$ más de probabilidad de tener un título universitario.

Los individuos que se encuentran actualmente estudiando, tiene un $30.6 \%$ menos de probabilidad de tener un título de bachiller, pues seguramente ya superaron, en su mayoría, este nivel de educación; así mismo, tienen un $17.11 \%$ y $20.38 \%$ menos de probabilidad de tener un título técnico o universitaria. Los que actualmente están trabajando, tienen un 8.8\% menos de probabilidad de tener un título de bachiller, y un $5.8 \%$ más de probabilidad de tener un título universitario. Quienes tienen computador en casa, tienen un $8.5 \%$ menos de probabilidad de tener un título de bachiller, y un $4.7 \%$ más de probabilidad de tener un título de técnico.

Las probabilidades predichas a partir del modelo muestran que la probabilidad de no tener ningún título académico es de $2.83 \%$, mientras que la probabilidad de tener un título de bachiller es la más alto, 65.8\%; la probabilidad de ser técnico es del $19.97 \%$, mientras que la probabilidad de ser profesional disminuye hasta llegar al 11.38\%.

En la tabla 7, se muestran las probabilidades de tener un determinado título académico de acuerdo con las diferentes categorías de cada una de las variables explicativas binarias. Por ejemplo, las estimaciones muestran que los hombres tienen una probabilidad del $7 \%$ de no tener ningún título, mientras que la probabilidad de las mujeres es del $4.8 \%$. Los hombres también tienen una mayor probabilidad de tener un título de bachiller $(62.29 \%)$ que las mujeres (57\%). Por otra parte, las mujeres tienen una probabilidad de $20.43 \%$ de tener un título técnico, que es mayor a la registrada por los hombres (17.8\%). Con respecto a los títulos universitarios, las mujeres (17.6\%) también tienen una mayor probabilidad que los hombres (12.7\%). 
Tabla 7

Efectos Marginales de las Variables Categóricas

\section{Título Obtenido}

\begin{tabular}{|c|c|c|c|c|}
\hline Variables & Ninguno & Bachiller & Técnico & Universitario \\
\hline \multicolumn{5}{|l|}{ Género } \\
\hline Hombre & $0.0704 * * *$ & $0.6229 * * *$ & $0.1786 * * *$ & $0.1279 * * *$ \\
\hline Mujer & $0.0488 * * *$ & $0.5708 * * *$ & $0.2043 * * *$ & $0.1760 * * *$ \\
\hline \multicolumn{5}{|l|}{ Zona } \\
\hline Urbano & $0.0567 * * *$ & $0.5952 * * *$ & $0.1940 * * *$ & $0.1540 * * *$ \\
\hline Rural & $0.0713 * * *$ & $0.6265^{* * *}$ & $0.1769 * * *$ & $0.1251 * * *$ \\
\hline \multicolumn{5}{|l|}{ Internet } \\
\hline Con Internet & $0.0505^{* * *}$ & $0.5845 * * *$ & $0.2015^{* * *}$ & $0.1632^{* * *}$ \\
\hline Sin Internet & $0.0651 * * *$ & $0.6210 * * *$ & $0.1831 * * *$ & $0.1306 * * *$ \\
\hline \multicolumn{5}{|l|}{ Computador } \\
\hline Con Computador & $0.0428 * * *$ & $0.5639 * * *$ & $0.2123 * * *$ & $0.1807^{* * *}$ \\
\hline Sin Computador & $0.0688 * * *$ & $0.6334 * * *$ & $0.1783 * * *$ & $0.1194 * * *$ \\
\hline \multicolumn{5}{|l|}{ Civil } \\
\hline Casado & $0.0559 * * *$ & $0.5931 * * *$ & $0.1948^{* * *}$ & $0.1560 * * *$ \\
\hline Soltero & $0.0584 * * *$ & $0.5995^{* * *}$ & $0.1917^{* * *}$ & $0.1502 * * *$ \\
\hline \multicolumn{5}{|l|}{ Jefe de Hogar } \\
\hline Si es Jefe & $0.0514 * * *$ & $0.5836 * * *$ & $0.1997 * * *$ & $0.1651^{* * *}$ \\
\hline No es Jefe & $0.0595 * * *$ & $0.6047 * * *$ & $0.1897 * * *$ & $0.1459 * * *$ \\
\hline \multicolumn{5}{|l|}{ Régimen } \\
\hline Contributivo & $0.0416 * * *$ & $0.5757 * * *$ & $0.2099 * * *$ & $0.1725^{* * *}$ \\
\hline Subsidiado & $0.0749 * * *$ & $0.6564 * * *$ & $0.1661 * * *$ & $0.1024 * * *$ \\
\hline \multicolumn{5}{|l|}{ Hijo } \\
\hline Es Hijo & $0.0537 * * *$ & $0.5807 * * *$ & $0.1991 * * *$ & $0.1663 * * *$ \\
\hline No es Hijo & $0.0610 * * *$ & $0.5993 * * *$ & $0.1904 * * *$ & $0.1491^{* * *}$ \\
\hline \multicolumn{5}{|l|}{ Desempleado } \\
\hline Desempleado & $0.0198 * * *$ & $0.4320 * * *$ & $0.2375^{* * *}$ & $0.3105^{* * *}$ \\
\hline No Desempleado & $0.0592 * * *$ & $0.6012 * * *$ & $0.1906 * * *$ & $0.1489 * * *$ \\
\hline \multicolumn{5}{|l|}{ Estudiante } \\
\hline Estudia & $0.1843 * * *$ & $0.7135^{* * *}$ & $0.0752 * * *$ & $0.0268 * * *$ \\
\hline No Estudia & $0.0315^{* * *}$ & $0.5892 * * *$ & $0.2112^{* * *}$ & $0.1679 * * *$ \\
\hline \multicolumn{5}{|l|}{ Tipo de Vivienda } \\
\hline Propia & $0.0518^{* * *}$ & $0.5814 * * *$ & $0.2024 * * *$ & $0.1662 * * *$ \\
\hline Arrendada & $0.0620 * * *$ & $0.6073 * * *$ & $0.1880^{* * *}$ & $0.1425^{* * *}$ \\
\hline \multicolumn{5}{|l|}{ Situación Laboral } \\
\hline Trabaja & $0.0410 * * *$ & $0.5726 * * *$ & $0.2083 * * *$ & $0.1780 * * *$ \\
\hline No Trabaja & $0.0685^{* * *}$ & $0.6423 * * *$ & $0.1728^{* * *}$ & $0.1162 * * *$ \\
\hline
\end{tabular}

Elaboración propia con base en datos de la GEIH (DANE, 2018)

Nota: $* * *, * *, *$ denotes $1 \%, 5 \%$, and $10 \%$ significance level, respectively.

Los individuos que viven en la zona urbana tienen mayor probabilidad de tener títulos de educación técnica y superior, mientras que los que viven en la zona rural, tienen mayor probabilidad de alcanzar un título de bachillerato y no tener ningún título, lo cual se debe a una cuestión de oferta educativa. Los que viven en la zona urbana tienen un $15.4 \%$ de probabilidad de tener un título de educación superior, mientras que para los que viven en la zona rural la probabilidad es de $12.5 \%$. 
Los jefes del hogar también tienen mayor probabilidad de tener algún título de educación técnica y universitaria. Los jefes de hogar tienen un $19.9 \%$ de probabilidad de tener un título técnico, y un $16.5 \%$ de probabilidad de tener un título universitario. Los individuos que pertenecen al régimen contributivo muestran un comportamiento similar, $17.2 \%$ y 20.9\% son las probabilidades de los individuos que pertenecen al régimen contributivo de tener un título universitario y técnico, respectivamente. Los individuos que pertenecen al régimen subsidiado presentan una probabilidad del $65.6 \%$ de tener un título de bachiller.

Los individuos que tienen vivienda propia tienen un $20 \%$ de probabilidad de tener un título técnico, y un $16.6 \%$ de tener un título universitario. Los que viven en casas arrendadas y en comodato, tienen una probabilidad del $60.7 \%$ de tener título de bachiller, y $6.2 \%$ de no tener ningún título. Las personas con computador en casa tienen un $21.2 \%$ de probabilidad de tener un título técnico, y un $18 \%$ de tener un título universitario. Así mismo, para los que tienen internet, la probabilidad de ser técnicos es del $20.1 \%$ y la de ser profesionales es del $16.3 \%$. Los que no tienen computador en casa, tienen un $63.3 \%$ de probabilidad de ser bachiller, y un $6.8 \%$ de no tener ningún título académico. Los que no tienen acceso a internet, tienen un $62.1 \%$ de probabilidad de ser bachiller, y un $6.5 \%$ de probabilidad de no tener ningún título académico.

Los individuos que están desempleado tienen un 31\% de probabilidad de tener un título universitario, y un $23.7 \%$ de tener un título técnico. Así mismo, los que están empleados, tienen un $17.8 \%$ de probabilidad de tener un título universitario, y un $20.8 \%$ de tener un título técnico. Las personas que están estudiando, tienen un $71.3 \%$ de probabilidad de tener un título de bachiller, y un $18.4 \%$ de no poseer ningún título académico.

Las estimaciones no muestran diferencias relevantes entre las probabilidades de individuos solteros y casados, y entre personas que son hijos del jefe del hogar. Variables explicativas como la edad (medida en años), las personas del hogar (medida en número de personas) y el estrato socioeconómico (medido de uno a seis), se pueden analizar marginalmente de manera más detallada de acuerdo a su unidad de medida. La tabla 8 muestra como la edad (efecto calculado a partir de los 10 hasta los 60 años, en grupos diez años), el número de miembros en el hogar (efecto calculado a partir de los 3 miembros hasta los 8) y el estrato socioeconómico (efecto calculado desde el estrato 1 al 6) afectan la probabilidad de tener un determinado título académico.

Tabla 8

Efectos marginales de las variables continuas

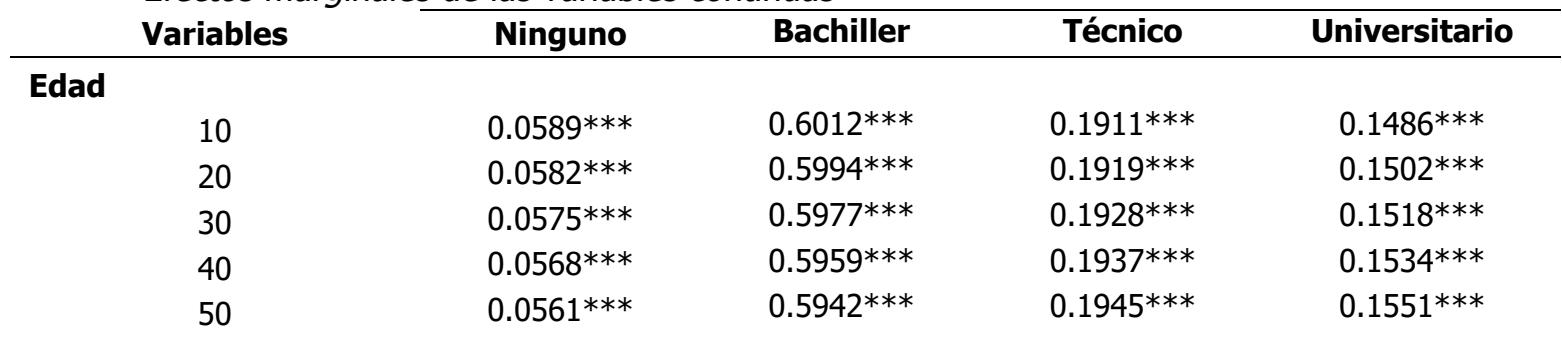




\begin{tabular}{|c|c|c|c|c|}
\hline 60 & $0.0554 * * *$ & $0.5924 * * *$ & $0.1954 * * *$ & $0.1567 * * *$ \\
\hline \multicolumn{5}{|l|}{ Estrato } \\
\hline 1 & $0.0736 * * *$ & $0.6481 * * *$ & $0.1708 * * *$ & $0.1073 * * *$ \\
\hline 2 & $0.0567 * * *$ & $0.6132 * * *$ & $0.1920 * * *$ & $0.1379 * * *$ \\
\hline 3 & $0.0430 * * *$ & $0.5720 * * *$ & $0.2109 * * *$ & $0.1739 * * *$ \\
\hline 4 & $0.0322 * * *$ & $0.5258 * * *$ & $0.2267 * * *$ & $0.2151^{* * *}$ \\
\hline 5 & $0.0237 * * *$ & $0.4764 * * *$ & $0.2384 * * *$ & $0.2612 * * *$ \\
\hline 6 & $0.0172 * * *$ & $0.4255^{* * *}$ & $0.2453 * * *$ & $0.3117 * * *$ \\
\hline \multicolumn{5}{|c|}{ Personas a Cargo } \\
\hline 3 & $0.0589 * * *$ & $0.5987 * * *$ & $0.1914 * * *$ & $0.1507 * * *$ \\
\hline 4 & $0.0629 * * *$ & $0.6077 * * *$ & $0.1868^{* * *}$ & $0.1424 * * *$ \\
\hline 5 & $0.0670 * * *$ & $0.6163 * * *$ & $0.1821 * * *$ & $0.1344 * * *$ \\
\hline 6 & $0.0713^{* * *}$ & $0.6245^{* * *}$ & $0.1772 * * *$ & $0.1267 * * *$ \\
\hline 7 & $0.0759 * * *$ & $0.6323 * * *$ & $0.1723 * * *$ & $0.1193 * * *$ \\
\hline 8 & $0.0806^{* * *}$ & $0.6396 * * *$ & $0.1673 * * *$ & $0.1122 * * *$ \\
\hline
\end{tabular}

Elaboración propia con base en datos de la GEIH (DANE, 2018)

Nota: $* * *, * *, *$ denotes $1 \%, 5 \%$, and $10 \%$ significance level, respectively.

Los resultados muestran que las variaciones unitarias en la edad no presentan grandes cambios en las probabilidades de acceder a cada uno de los niveles de títulos analizados. En los niveles técnico y universitario, la probabilidad de tener un título aumenta ligeramente a medida que aumenta la edad; una persona con 20 años de edad presenta una probabilidad de tener un título universitario del $15.02 \%$, mientras que una persona con 60 años, presenta una probabilidad del $15.67 \%$. En bachillerato, así como en las personas que no tienen ningún título académico, por el contrario, las probabilidades de tener un título disminuyen ligeramente a medida que aumenta la edad; una persona con 20 años de edad presenta una probabilidad de tener un título de bachiller de $59.9 \%$, mientras que, para un individuo con 60 años, la probabilidad es de $59.7 \%$.

Con respecto al número de personas en el hogar, a media que aumenta el número de personas, aumenta la probabilidad de no tener ningún título académico, así como la probabilidad de tener un título de bachiller. Un individuo que habita en un hogar con 3 personas presenta una probabilidad de tener un título de bachiller de $59.8 \%$, mientras que, en un hogar con 8 miembros, la probabilidad asciende a $63.9 \%$. Por el contrario, el número de miembros del hogar presenta una relación inversa con la probabilidad de tener un título técnico y universitario. Un individuo que habita en un hogar con 3 personas presenta una probabilidad de tener un título universitario de $15 \%$, mientras que, en un hogar con 8 miembros, la probabilidad disminuye hasta llegar a $11.2 \%$.

En los niveles de educación técnica y universitaria, se presenta una relación positiva con el estrato socioeconómico, mientras que en los niveles de bachiller y sin título universitario dicha relación es negativa. Un individuo de estrato 1 tiene un $10.7 \%$ de probabilidad de tener un título universitario, mientras en una persona de estrato 6 , dicha probabilidad aumenta hasta llegar al $31.1 \%$. En los técnicos, la probabilidad pasa de $17 \%$ a $24.5 \%$ en 
los mismos estratos analizados. En el caso de los bachilleres ocurre lo contrario, un individuo de estrato 1 tiene un $64.8 \%$ de probabilidad de ser bachilleres, mientras que una persona del estrato 6 presenta una probabilidad del $42.5 \%$.

\section{Tabla 9}

Comparación de pronóstico del modelo

\begin{tabular}{ccc} 
& \multicolumn{2}{c}{$\%$} \\
\hline Título & Pronósticos & Datos reales \\
\hline Ninguno & 5.67 & 5.46 \\
Bachiller & 60.57 & 58.48 \\
Técnico & 18.68 & 19.75 \\
Universitario & 15.09 & 16.29 \\
\hline propia con base en datos de la GEIH (DANE, 2018)
\end{tabular}

Elaboración propia con base en datos de la GEIH (DANE, 2018)

El modelo presenta un buen pronóstico. Mientras en los datos reales se observa que el $5.46 \%$ no tienen ningún título académico, el modelo pronostica que el $5.67 \%$ de los individuos no tendrían ningún nivel académico. Con respecto a los bachilleres, los datos reales corresponden al $58.48 \%$, mientras que los datos pronosticado se ubica en $60.57 \%$. En el nivel técnico, el modelo pronostica que el $18.68 \%$ del total de personas quedaría clasificada como técnica, los datos reales por su parte, alcanza el $19.75 \%$. Finalmente, el $16.29 \%$ del total de individuos de la base de datos presentan título universitario, el modelo pronostica que dicha cifra sería del $15.09 \%$.

\section{Discusiones y conclusiones}

Tener un título académico en Colombia, especialmente a nivel técnico y universitario, se ha convertido en una tarea cada vez más difícil. Más de la mitad de la población colombiana, apenas les alcanza para tener un título de bachiller, y 1.6 de cada 10 personas presentan un título universitario.

La edad es sin duda una variable que afecta la probabilidad de tener algún título académico. En los niveles de bachiller y ningún título, su efecto es negativa, es decir, a mayor edad es menos probable que alcancen estos niveles, mientras que, en los niveles técnico y universitario, su efecto es positivo, a mayor edad es más probable que alcancen dichos títulos. Esto resulta lógico, pues a mayor edad, es más probable que el individuo haya alcanzado el máximo nivel posible de educación y por esto presente menos probabilidad de tener niveles bajos de educación, y mayor probabilidad de tener niveles altos. 
Las mujeres están mejor capacitadas que los hombres, pues presentan mayores probabilidades de tener un título técnico y universitarios, y menor probabilidad de alcanzar títulos de menos nivel, en comparación con los hombres. Esta situación se viene presentando desde hace años en Colombia, sin embargo, aún no se refleja en el mercado laboral, pues la tasa de desempleo femenina es mayor que la tasa de desempleo masculino ${ }^{3}$, y las condiciones laborales, incluido el salario, aún presentan preferencias a favor de los hombres.

La zona de residencia es un aspecto que afecta la oferta educativa. En la zona rural, se presenta una mayor probabilidad de tener un título bachiller o ningún título, mientras que en la zona urbana es más probable encontrar individuos con formación técnica y profesional. Es un tema de acceso a la oferta. Del mismo modo, un número mayor de personas en el hogar disminuye la probabilidad de alcanzar un nivel académico más alto, esto se explica principalmente por las condiciones socioeconómicas, un mayor número de personas limita el acceso de algunos miembros a la educación técnica o universitaria, pues los recursos económicos se deben distribuir en un número mayor de personas.

Las condiciones socioeconómicas sin duda determinan el acceso a la educación, especialmente en niveles más altos, pues en los niveles técnicos (con excepción del Sena) y universitarios, se debe pagar para acceder a ellos. De nuevo, mayores estratos socioeconómicos e individuos con vivienda propia presentan mayor probabilidad de acceder a niveles de educación más altos; mientras que, en los menores estratos e individuos con viviendas en arriendo, presentan mayor probabilidad de no tener ningún título académico, o tener un título de bachiller; menores condiciones socioeconómicas limitan el acceso a niveles de educación más altos.

Los jefes del hogar y los casados presentan una mayor probabilidad de acceder a títulos de educación más altos, que los que no son jefes de hogar y los solteros. Mayores responsabilidades implica buscar posibilidades de acceder a niveles de ingresos más altos, y la educación es sin duda la mejor herramienta. Sin embargo, una mayor educación no garantiza que los individuos estén trabajando, pues de acuerdo con los resultados encontrados, los desempleados presentan mayor probabilidad de tener título de educación técnico y universitario, y menor probabilidad de tener título de bachiller, o ningún título.

Las herramientas tecnológicas también presentan un importante impacto sobre la probabilidad de tener un determinado título de educación. Los individuos que tienen computador y acceso a Internet, presentan mayor probabilidad de alcanzar niveles mayores de educación, pues teóricamente los avances tecnológicos facilitan el desarrollo de distintos procesos educativos.

\footnotetext{
${ }^{3}$ De acuerdo con cifras del Dane, en el segundo trimestre del 2019, la tasa de desempleo en Colombia fue de $10.2 \%$, la tasa de desempleo de los hombres alcanzó $8.1 \%$, mientras que en las mujeres ascendió al $13.1 \%$.
} 
Finalmente, se puede evidenciar que en la informalidad se encuentran especialmente individuos con menores de niveles de educación, mientras que en la formalidad trabajan personas con mayores niveles de educación. Esto se demuestra al observar el efecto de la variable contributivo (que indica si el individuo pertenece al régimen contributivo de salud), que es un indicador claro de formalidad; quienes pertenecen al sector contributivo, tienen una mayor probabilidad de tener un título técnico y universitario, mientras que quienes pertenecen al sector subsidiado, presentan mayor probabilidad de acceder a un título de bachiller o no tener ningún nivel de educación.

\section{Referencias}

Becker, G. (1975). Human Capital: A Theoretical and Empirical Analysis, with Special Reference to Education. National Bureau of Economic Research.

Becker, G. (1993). Human capital: a theoretical and empirical analysis with special reference to education. University of Chicago Press.

Benhabib, J. \& Spiegel, M. M. (1994). The role of human capital in economic development evidence from aggregate cross-country data. Journal of Monetary economics, 34(2), 143-173.

Cameron, C. \& Trivedi, P. (2005). Microeconometrics Methods and Applications. Cambridge University Press.

Cameron, S. V. \& Hechman, J. J. (1998). Life Cycle Schooling and Dynamic Selection Bias: Models and Evidence for Five Cohorts of American Males. The Journal of Political Economy, 106(2), 262-333. https://www.journals.uchicago.edu/doi/10.1086/250010

Cameron, S. V. \& Taber, C. J. (1994). Evaluation and Identification of Semiparametri Maximum Likelihood Models of Dynamic Discrete Choice. Manuscript Chicago.

Cerquera, O., Gomez, C. y Cano, J. (2016). Factores asociados al logro académico en regiones desarrolladas y en desarrollo de Colombia: una aproximación a partir de un modelo de combinación de corte transversal. Revista Historia de la Educación Colombiana 19(19), 167-201.

CEPAL. (2019). Perspectivas económicas de América Latina 2019: Desarrollo en transición. OECD Publishing. https://doi.org/10.1787/g2g9ff1a-es

Connelly, J., Philbrick, J., Smith, G., Kaiser, D., \& Wymer, A. (1989). Health perceptions of primary care patients and the influence on health care utilization. Supplement to Medical Care, (27), 99-109. https://doi.org/10.1097/00005650-198903001-00009

DANE. (2018). Gran Encuesta Integrada de Hogares - GEIH - 2018. 


\section{http://microdatos.dane.gov.co/index.php/catalog/547}

Ferreyra, M. (2017). La educación superior en América Latina y el Caribe. Grupo Banco Mundial.

Fundación Empresarios por la Educación. (Abril de 2019). Informe de gestión. https://fundacionexe.org.co/wp-content/uploads/2020/06/Informe-gestion-.pdf

Giovagnoli, P. I. (2002). Determinantes de la deserción y graduación universitaria: Una aplicación utilizando modelos de duración. [Tesis de Maestría, Universidad Nacional de La Plata].

González, R. M. (1989). Análisis de las causas del fracaso escolar en la Universidad Politécnica de Madrid. Ministerio de Educación Cultura y Deporte, Centro de Investigación y Documentación Educativa.

Hanushek, E. A. (1986). The economics of Schooling. Journal of Economic Literature, 24(3), 1141-1171.

Hernández, R., Cuevas, A., Leal, B. y Mendoza, C. (2016). Enseñanza-aprendizaje de ciencia e investigación en educación básica en México. Revista electrónica de investigación educativa, 18(3), 187-200.

Hernández, R., Fernández, C., y Baptista, M. (2010). Metodología de la Investigación. Mc Graw Hill.

Kerlinger, F. (2002). Investigación del Comportamiento. MC Graw Hill.

Long, J., \& Freese, J. (2001). Regression Models for Categorical Dependent Variables Using Stata. Stata Press.

Lucas, R. (1988). On the mechanics of economic development. Journal of monetary economics, 22(1), 3-42.

Mankiw, G. (1992). A contribution to the empirics of economic growth. The quarterly journal of economics, 107(2), 407-437.

Mendoza, L., Mendoza, U. y Romero, D. (2014). Permanencia académica: una preocupación de las instituciones de educación universitaria. Escenarios, 12(2), 130-137. https://doi.org/10.15665/esc.v12i2.320

OCDE. (2016). La naturaleza del aprendizaje: Usando la investigación para inspirar la práctica. OCDE, OIE-UNESCO, UNICEF.

OCDE. (2019). Education at a Glance. OECD Indicators. 
Osorio, A., Bolancé, C. y Castillo, M. (2012). Deserción y graduación estudiantil universitaria: una aplicación de los modelos de supervivencia. Revista iberoamericana de educación Superior, $3(6)$,

31-57. https://doi.org/10.22201/iisue.20072872e.2012.6.55

Persoglia, L., Carella, L. y Solari, E. (2017). Rendimiento académico y características socioeconómicas de graduados. Revista Ciencia, docencia y tecnología, 28(54), 235251.

Romer, P. (1986). Increasing Returns and Long-Run Growth. Journal of political Economy, 94(5), 1002-1037.

Romer, P. (1989). Increasing returns and new developments in the theory of growth. NBER Working Paper Series, (3098), 2-40.

Williams, R. (2006). Generalized Ordered Logit/ Partial Proportional Odds Models for Ordinal Dependent Variables. The Stata Journal, 6(1), 58-82. https://doi.org/10.1177/1536867X0600600104

\section{Agradecimientos}

Este artículo es el resultado del proyecto de investigación Determinantes de la Calidad de la Educación en Colombia, desarrollada al interior del grupo de investigación IGUAQUE de la Facultad de Economía y Administración de la Universidad Surcolombiana. Este proyecto fue financiado mediante la convocatoria por la Vicerrectoría de Investigación y Proyección Social de la Universidad Surcolombiana, en el marco de las Convocatorias de Investigación de Menor Cuantía. 